This is an Accepted Manuscript of an article published by Taylor \& Francis in Vehicle System Dynamics on the $2^{\text {nd }}$ of November 2016, available online:

http://www.tandfonline.com/doi/full/10.1080/00423114.2016.1251598 


\title{
Design of Tyre Force Excitation for Tyre-Road Friction Estimation
}

*Anton Albinsson (Corresponding author), *Fredrik Bruzelius, *Bengt Jacobson, *Jonas Fredriksson

\author{
*Chalmers Technical University \\ 41296 Gothenburg Sweden \\ Phone: $+46(0) 317721000$ \\ E-mail: anton.albinsson@chalmers.se, \\ fredrik.bruzelius@chalmers.se, \\ bengt.jacobson@chalmers.se \\ jonas.fredriksson@chalmers.se
}

\begin{abstract}
Knowledge of the current tyre-road friction coefficient is essential for future autonomous vehicles. The environmental conditions, and the tyre-road friction in particular, determine both the braking distance and the maximum cornering velocity and thus set the boundaries for the vehicle. Tyre-road friction is difficult to estimate during normal driving due to low levels of tyre force excitation. This problem can be solved by using active tyre force excitation. A torque is added to one or several wheels in the purpose of estimating the tyre-road friction coefficient. Active tyre force excitation provides the opportunity to design the tyre force excitation freely. This study investigates how the tyre force should be applied to minimize the error of the tyre-road friction estimate. The performance of different excitation strategies were found to be dependent on both tyre model choice and noise level. Furthermore, the advantage with using tyre models with more parameters decreased when noise was added to the force and slip ratio.
\end{abstract}

Keywords: Tyre-road friction estimation, Vehicle dynamics, State estimation, Active tyre force excitation, Optimization,

\section{Acknowledgements}

This work was supported by VINNOVA (the Swedish Governmental Agency for Innovation Systems) under grant number 2012-03658. 


\section{Introduction}

In strive for autonomous vehicles modern passenger cars are being equipped with an increasing number of Advanced Driver Assistance Systems (ADAS). Future fully autonomous vehicles, level 5 autonomous vehicles as defined in [1], must be able to handle all aspects of driving for all roadway and environmental conditions manageable by a human driver. The environmental conditions and the tyre-road friction in particular, determine the braking distance and the maximum cornering velocity. Without any knowledge of the tyre-road friction the worst-case scenario would have to be assumed, implying a very conservative drive strategy for autonomous vehicles. This could result in poor customer acceptance since there will be situations where it is obvious to the passengers/driver that the safety margins are unreasonably large for the circumstances. A conservative drive strategy could potentially also decrease the traffic flow and create less acceptance from other vehicles on the road. Adapting the driving strategy to the current environmental conditions is therefore important for autonomous vehicles. Lower level of autonomous driving and active safety systems can also benefit from real-time information about the current tyre-road friction coefficient. Autonomous Emergency Braking (AEB) could for instance be improved by adapting the intervention thresholds based on an estimated friction coefficient.

There exist many, fundamentally different, approaches to estimate the current environmental conditions. A simple example is the warning message in the instrument cluster when the ambient temperature is close to or below water freezing temperature. Although this information is useful for the driver, the ambient temperature does not give a direct indication of the current tyre-road friction coefficient. Hence, for active safety systems and autonomous vehicles more accurate information is required. Friction estimation methods in general can be divided into two different categories, cause- and effect-based methods $[2,3]$. Cause-based approaches, as the name suggests, identify the environmental causes for different tyre-road friction coefficients such as, ambient temperature, water on the road, snow, ice etc. The effect-based approaches measure the effects of the current road surface on the vehicle response to identify a tyre-road friction coefficient. Optical systems are commonly used for cause-based approaches, see for example [4]. These systems can detect the road surface ahead of the vehicle and thus provide a short preview of the upcoming road.

Effect-based approaches can only detect the current friction coefficient. However, the consequence of this can be resolved by introducing cloud-based communication between vehicles, see for instance the project Road Status Information (RSI) [5]. Effect-based approaches also have the advantage of estimating the actual tyre-road friction coefficient given by the current tyre-road combination. The friction coefficient can vary on the same road-surface for different tyres depending on the properties of the tyre. It should also be noted that the same tyre on the same class of surface (snow, ice, asphalt etc.) can have different friction coefficients depending on the structure of the surface and other ambient conditions [6]. This makes it difficult to determine the friction coefficient accurately by only classifying the road surface. When cloud-based services are used to share friction information between vehicles, effectbased friction estimation approaches will provide friction information for the tyres that are fitted to the measuring vehicle. This mean that the effect-based friction estimators cannot provide highly accurate preview information and predict the exact future friction coefficient for the vehicle. However, friction coefficient variations in the same class of road surface can still be possible to capture on average with several measuring vehicles available.

A drawback with effect-based approaches is that they normally require large tyre excitation (large tyre forces) to achieve accurate friction estimates [7-11]. Studies that investigate if the 
maximum road-friction coefficient can be estimated from only the slip stiffness of the tyre-road combination have been made, see e.g. $[12,13]$. This would reduce the required tyre excitation to levels which can be found during normal driving. Although the road surface influence on the slip stiffness has been shown, no straightforward correlation between the slip stiffness and the maximum road friction coefficient has been shown in literature. Furthermore, the slip stiffness is sensitive to other parameters than the road surface, see [14]. More information than the slip stiffness is therefore needed to determine the friction coefficient. However, different road surfaces may be identified using the slip stiffness with a-priori information about the tyres and the road surfaces that the vehicle will operate on. In practice then, with unknown tyres fitted to the vehicle and at least some road surfaces that are not known a-priori, the requirement of large tyre excitation still remains.

The requirement of high excitation implies that the tyre-road friction coefficient cannot be estimated during normal driving, except for very slippery road conditions. Possibilities to estimate the friction coefficient are thus scarce when relying purely on the driver input in dayto-day driving conditions. It has been previously suggested in literature to use active tyre force excitation where torques are actively applied to the wheels in the sole purpose to estimate the tyre-road friction coefficient [15-17]. The authors have previously published a proof-of-concept where this method was implemented and evaluated in a real vehicle, see [18]. The basic concept of active tyre force excitation is to achieve large opposite tyre forces on the front and the rear axle, while maintaining the intended acceleration of the driver. This is possible by adding a propulsion torque on the front axle while using the friction brake on the rear axle to maintain the desired acceleration level. The level of excitation, the location and time of the friction estimation, can thus be controlled. Naturally, it has to be ensured that the stability of the vehicle is guaranteed and that the wear, energy consumption and comfort are acceptable.

With the possibility to control the tyre forces freely, and thus also the data used for friction estimation, a new degree of freedom is available when developing the tyre-road friction estimator. Instead of relying on the data from the driver inputs only, it is possible, within the limitations of the actuators, to design the measurements. This paper exploits the new degree of freedom to excite the tyre forces to improve the quality of the estimated friction. Different strategies are investigated and compared, including ones derived from optimization. Focus is put on the influence of modelling errors of the tire model, with three commonly used models in the investigation. Measurements from two different surfaces are used as reference, representing the actual tyre behaviour. 


\section{Method}

This section describes the different tyre models used in the investigation and the optimization of the excitation. The excitations are evaluated for six different tyre-road combinations. Only pure longitudinal slip is considered since the lateral tyre forces can be neglected in the motivating problem, see [18]. In a real vehicle, the intervention may be performed when small lateral tyre forces are present as well. Furthermore, the tyre dynamics has been neglected since it is assumed that the active tyre force excitation will be done slow enough to neglect the tyre relaxation. This is motivated by the aim of the study, to understand which measurement points to choose without making the problem unnecessarily complicated. A batch estimation strategy requires that the friction coefficient is estimated after the intervention. This strategy is suitable in combination with active tyre force excitation since the intervention is well-defined and time limited. However, this paper does not define when the estimator should be switched off for a real intervention. The batch strategy also allows the estimator to take all the measurement points into consideration simultaneously. A flow chart of the optimization method can be seen in Figure 1. The details of the optimization method are described in the other sections in this chapter.

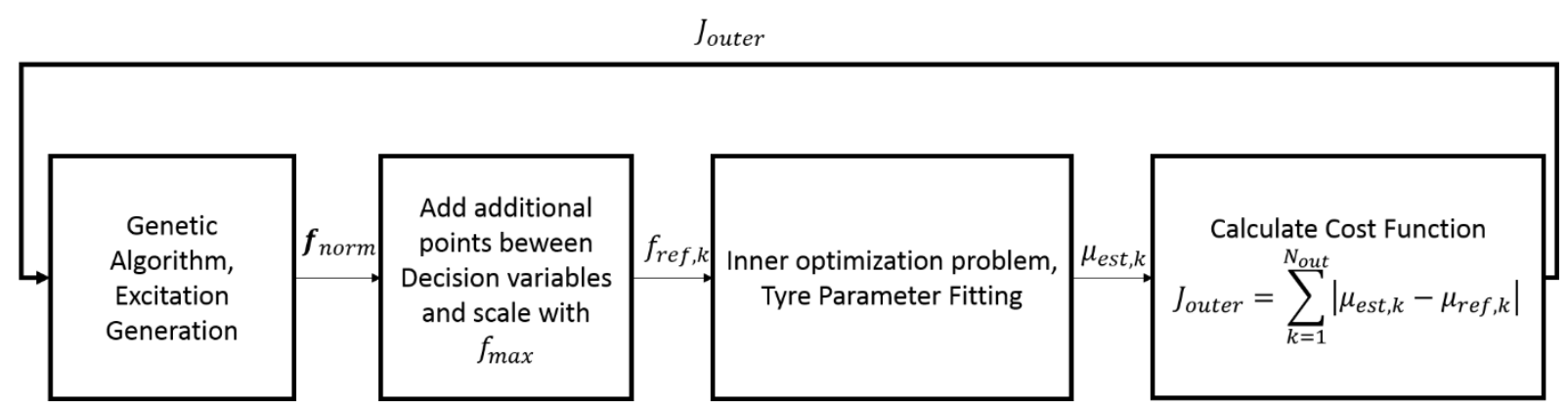

Figure 1, Flow chart of optimization method

The optimization is performed for all tyre-road combinations simultaneously in order to study the influence of modelling errors and to find a generic estimation strategy which is valid for several road surfaces. When implemented in a real vehicle the road surface is unknown and the estimation strategy should thus minimize the estimation error for all road surfaces. The optimization is also performed on asphalt and gravel separately to compare the differences in excitation strategy. The main purpose of the excitation optimization was to investigate any general trends in the optimized excitation that could serve as inspiration to other excitation strategies that are implementable in a real vehicle and to use the optimized excitations as benchmarks to which other excitation strategies could be compared.

\subsection{Reference Tyre Characteristics}

Due to the loose surface on gravel, the slip stiffness tends to be lower and the peak less distinguished as compared to wet asphalt. By using two different road surfaces a more general excitation can be obtained that minimizes the friction estimation error for several different tyre and road surface combinations. The measurements, which are the same measurements that were used in [8], are performed with the mobile tyre testing rig BV12. A negative torque is applied to the wheel and decreased until wheel lock. The measurement signals used in this paper are the longitudinal force $F_{x}$, the vertical force $F_{z}$, the rotational velocity of the wheel $\omega$ and the longitudinal velocity of the wheel centre $v_{x}$.

The reference measurements were filtered and the slip and force offsets removed so that the slip is zero at zero longitudinal force. Furthermore, in order to optimize the excitation, the tyre force was normalized with both the vertical force, 


$$
f=\frac{F_{x}}{F_{z}}
$$

and the maximum normalized force,

$$
f_{\text {norm }}=\frac{f}{f_{\max }}
$$

The second normalization, Equation (2), was done to have decision variables with the same relative excitation levels for the different tyre-road surface combinations in the optimization problem, see Figure 2. The oscillations in the measured tyre characteristics at larger slip values are due to oscillations in the vertical force measurements. Even though the tyre characteristics are averaged over several measurements, it is difficult to get a large number of measurement points at slip values beyond the peak force. The purpose of a friction estimator is to estimate the friction level before the maximum force is reached, hence only measurement points below the peak force were considered in this paper.
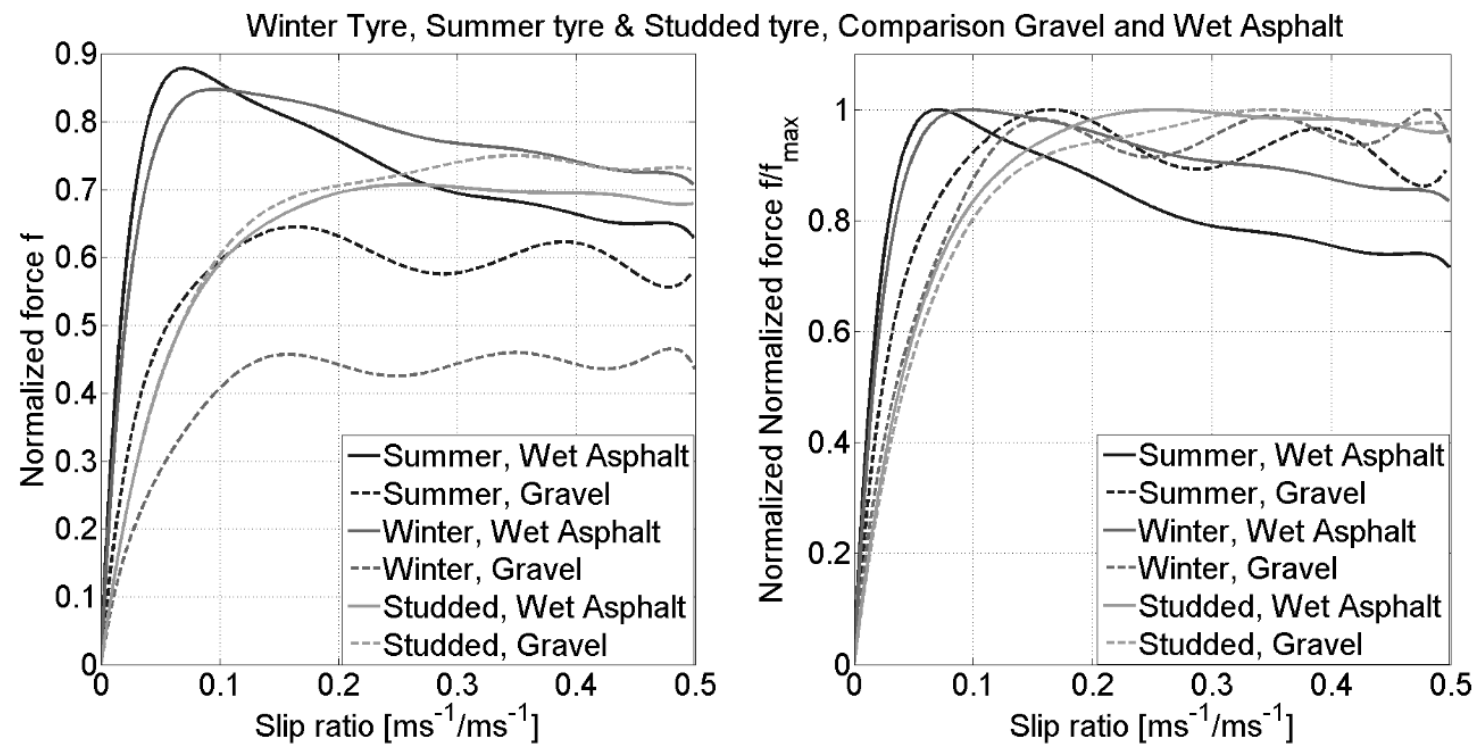

Figure 2, Measured and filtered normalized tyre force $f$ and normalized normalized tyre force $f_{\text {norm }}$ vs longitudinal slip ratio for the different tyre-road combinations.

The reference tyre characteristics are implicitly assumed to be representative for the tested tyres and surfaces. Naturally, the test method, equipment and the environmental conditions influence the test results. Furthermore, these tyre-road characteristics are not valid for other asphalt and gravel surfaces. Although the reference tyres do not describe the tested tyre perfectly, they represent a variety of tyre-road combinations, thus allowing for a more general formulation of the results and conclusions. The slip ratio $\sigma_{x}$ used throughout the paper, is defined as

$$
\sigma_{x}=\frac{v_{x}-R \omega}{R \omega}
$$

\subsection{Evaluated Tyre Models}

Two of the models chosen for evaluation are based on the brush tyre model but with different pressure distributions, parabolic (Equation (4)) and constant pressure distribution (Equation (5)) 


$$
f\left(\sigma_{x}\right)=\left\{\begin{array}{cl}
-C_{\sigma_{x}} \sigma_{x}+ & \frac{C_{\sigma_{x}}^{2} \sigma_{x}\left|\sigma_{x}\right|}{3 \mu}-\frac{C_{\sigma_{x}}^{3} \sigma_{x}^{3}}{27 \mu^{2}} \\
-\operatorname{sign}\left(\sigma_{x}\right) \mu F_{z} & \text { for }\left|\sigma_{x}\right| \leq \frac{3 \mu}{C_{\sigma_{x}}}
\end{array}\right.
$$

The brush model with constant pressure distribution is often referred to as the Dugoff tyre model [19]. It is given by,

$$
\begin{array}{cl}
f\left(\sigma_{x}\right)=\left\{\begin{array}{cl}
C_{\sigma_{x} \sigma_{x}(2-\lambda) \lambda} & \text { for }|\lambda|<1 \\
C_{\sigma_{x}} \sigma_{x} & \text { else }
\end{array}\right. \\
\qquad \lambda=\frac{\mu}{2\left|C_{\sigma_{x}} \sigma_{x}\right|}
\end{array}
$$

These tyre models have two parameters for pure longitudinal slip, the slip stiffness $C_{\sigma_{x}}$ and the friction coefficient $\mu$ and are not adaptable to different curvatures of the slip-force curve. In contrast the Magic formula has four parameters and thus additional freedom to adapt the resulting tyre characteristics [20],

$$
f\left(\sigma_{x}\right)=D \sin \left(C \tan ^{-1}\left(B \sigma_{x}-E\left(B \sigma_{x}-\tan ^{-1}\left(B \sigma_{x}\right)\right)\right)\right)
$$

The $C$ parameter in the magic formula mainly affects the decrease in force after the peak of the tyre characteristics and the E parameter mainly affects the shape near the peak. Two different variations of the Magic formula are evaluated and compared, one where the $C$ parameter is fixed to one and another model where the $C$ parameter is allowed to vary in the range from 1 to 1.6. The effect of adding additional parameters is thus evaluated. The range of the other tyre parameters has to be constrained to make sure that the resulting tyre characteristics are realistic and to aid the optimization problem in estimating the tyre parameters. The parameter ranges can be seen in table 1 .

Table 1, Tyre Parameter Constraints

\begin{tabular}{|l|l|c|l|}
\hline$C_{\sigma_{x}}$ & {$[1,200]$} & $\mathrm{B}$ & {$[1,200]$} \\
\hline$\mu$ & {$[0.05,1.5]$} & $C$ & {$[1,1]$ or $[1,1.6]$} \\
\hline$D$ & {$[0.05,1.5]$} & $E$ & {$[-1.5,1]$} \\
\hline
\end{tabular}

\subsubsection{Parametric sensitivity analysis}

A sensitivity analysis was performed for the summer tyre to understand in which region data should be sampled to estimate the different tyre parameters, see Figure 3 and 4 . The variations have been normalized so that the maximum deviation corresponds to 0.1 in normalized tyre force $f$. The grey areas are derived from the partial derivatives of the force with respect to the different parameters at that slip ratio value. Note that the figures show the first order variation around the nominal value which describes the nominal deviation in each slip point and not how the curve is affected globally by the different parameters. Figure 4 clearly shows the difference between the influence of the slip stiffness and the friction parameter for the brush model and the Dugoff model. The results for the Magic formula indicate that the B, C and E parameter all have similar effect on the tyre characteristics but at different slip levels. Note that the E 
parameter does not affect the linear tyre region in the Magic formula as opposed to the B and C parameters.

The sensitivity analysis can explain part of the performance of the optimization procedure. It is important to remember that these figures only indicate at which force level a certain parameter should be estimated provided that there are no modelling errors. If modelling errors are present, it may be beneficial to avoid a certain excitation level in order to avoid that areas with large modelling errors influence the result. Naturally, with real test data, modelling errors are present and depending on the size of these errors, the optimized excitation will either avoid or seek out the areas where the partial derivatives with respect to the unknown parameters are large. Figure 3 and 4. also show why a large excitation is required to determine the friction coefficient accurately. At low tyre forces, there is not enough information to determine the peak value. This information from the sensitivity analysis could potentially be included in the optimization algorithm to actively seek out or avoid utilization levels with large parameter gradients depending on the size of the modelling error. In real-time, the information could be used to give different weights to the measurement points provided that a recursive tyre parameter estimator is used. The main purpose to include the sensitivity analysis in this study was to indicate why the genetic algorithm used in the optimization procedure prefer some utilization levels.
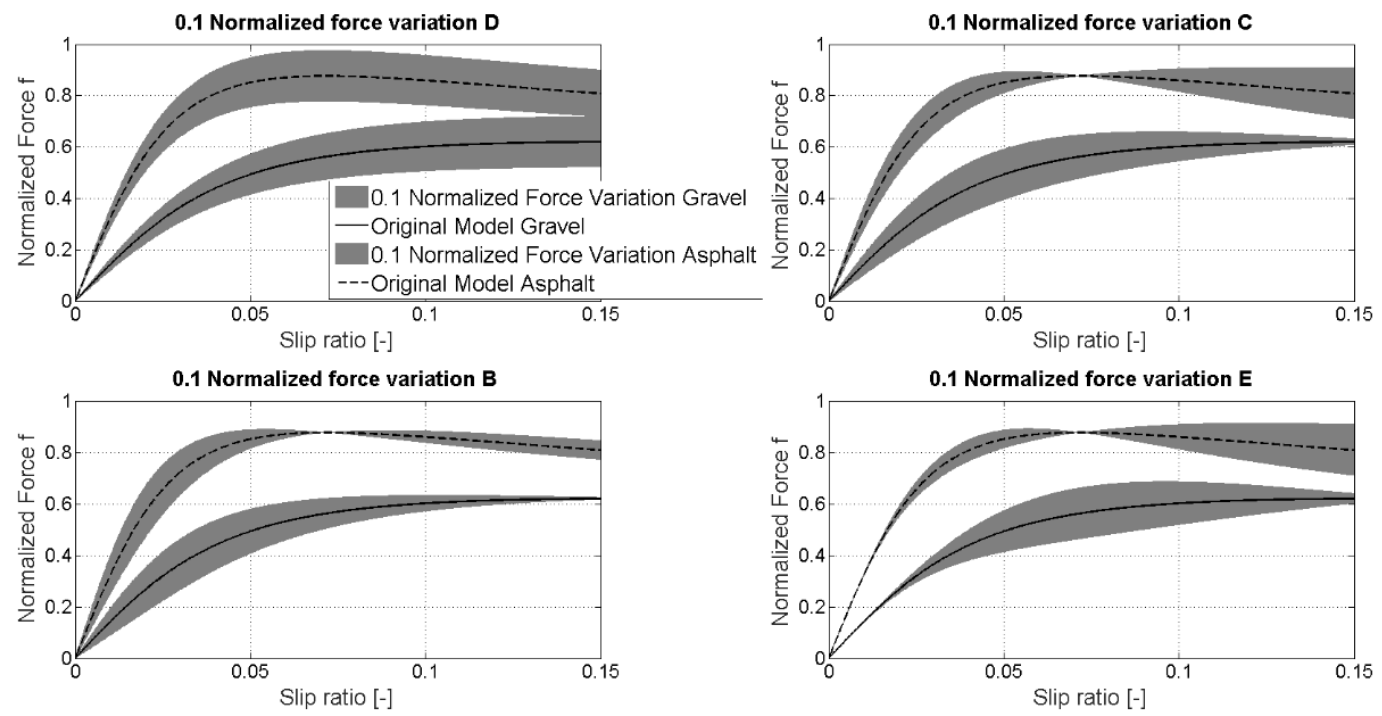

Figure 3, Parameter sensitivity Magic formula, 0.1 normalized force variation. 

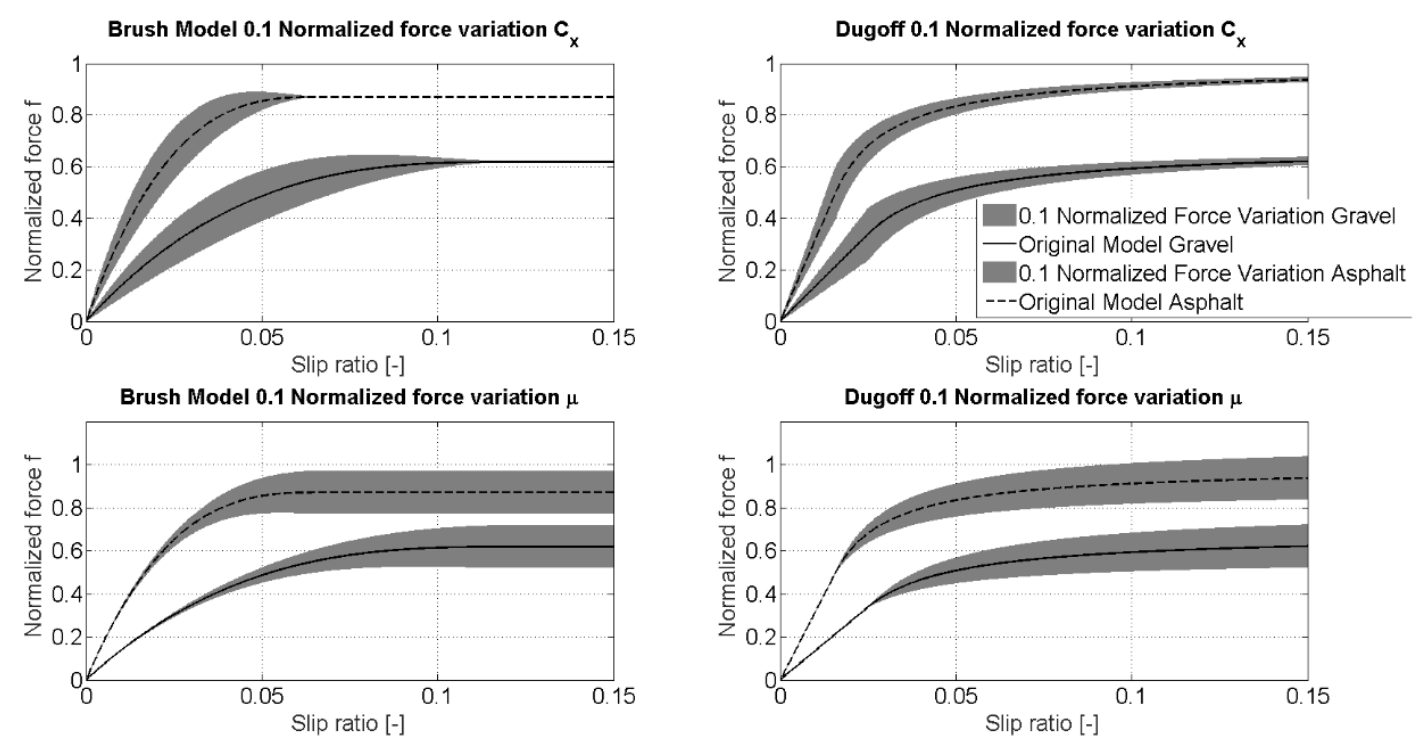

Figure 4, Parameter sensitivity Brush model and Dugoff model, 0.1 normalized force variation.

\subsection{Optimization Problem}

This section describes and formulates the optimization problem. The objective of the optimization is to find the best excitation that minimizes the friction estimation error. The optimization problem is split into two different layers of interconnected optimization problems, inner problems and an outer problem. The inner problems consist of the tyre parameter fitting to a given tyre force excitation, Equation (12). In a real-world application this inner optimization problem can be replaced by a recursive estimator. However, to study the optimized excitation independently of the estimator approach, batch optimization problems were formulated for the inner layer. The tyre parameters are thus found through optimization for each tyre-road combination using all the available data. The goal of the outer optimization problem is to minimize the friction coefficient estimation errors of the inner problems, i.e. the difference between the measured and estimated maximum friction coefficients, see Equation (7).

$$
J_{\text {outer }}=\sum_{k=1}^{N_{\text {out }}}\left|\mu_{\text {est }, k}-\mu_{\text {ref }, k}\right|
$$

The force in the inner optimisation problems $f_{r e f, k}$ (Equation (12)) corresponds to the normalized force $f$ (Equation (1)) and $\mu_{r e f, k}$ corresponds to the maximum normalized force $f_{\text {max }}$ in Equation (2) for the tyre-road combination $\mathrm{k}$. As seen from Equation (6), the cost function of the outer optimization problem is defined as the sum of the absolute errors of the friction estimates for the six different road-tyre combination. By choosing the absolute error instead of the square error of each estimate, large estimation errors have a lower weighting. Hence, the risk of finding a minimum that mainly minimizes the largest friction estimation error is reduced. The optimized force excitation, normalized with the vertical force and $f_{\text {max }}$ according to Equation (2), is found as,

$$
\boldsymbol{f}_{\text {norm }}^{*}=\arg \min _{\boldsymbol{f}_{\text {norm }}} J_{\text {outer }}
$$

with the constraints 


$$
\begin{gathered}
0<\boldsymbol{f}_{\text {norm }}(i)<f_{\text {norm,max }} \text { for } i=2,3 \ldots N \\
\boldsymbol{f}_{\text {norm }}(1)=0
\end{gathered}
$$

The tyre models that are used to estimate the tyre parameters do not perfectly describe the measured tyre characteristics and modelling errors will therefore be present. The optimized excitation for a single tyre-road combination will be influenced by these modelling errors. Hence, by only selecting a few different excitation levels, the friction estimation error can be very small for a single tyre-road combination but the obtained excitation would not perform as well for other surfaces or tyres. The optimization of the excitation was thus done to minimize the friction estimate error for all six different tyre-road surface combinations simultaneously. By using six different road-tyre combinations the effect of modelling errors in the tyre model will be captured in the optimized excitation. The friction coefficient in the inner optimization problem is estimated by minimizing the force error between the model and the chosen excitation points, Equation (11-15) where

$$
\mu_{\text {est }, k}=\arg \min _{\boldsymbol{\theta}_{\text {inn }}} J_{\text {inner }}
$$

with,

$$
\begin{gathered}
J_{\text {inner }}=\sum_{m=1}^{N_{\text {inn }}}\left(f_{\text {ref }, k}(m)-f\left(\boldsymbol{\theta}_{\text {inn }}, \sigma_{\text {ref }, k}(m)\right)\right)^{2} \\
f_{\text {ref }, k}=\left[f_{r e f, k, 1} f_{r e f, k, 2} \ldots f_{r e f, k, N}\right] \\
\sigma_{r e f, k}=f_{\text {tyre }}^{-1}\left(f_{\text {ref }, k}\right)
\end{gathered}
$$

where $f_{r e f, k}$ is the normalized force (with vertical force) for each road-tyre combination and $\boldsymbol{\theta}_{i n n}$ are the unknown tyre parameters. With $\mu_{r e f, k}$ denoting the reference friction coefficient from the measurements the different components in $f_{r e f, k}$ can be expressed as in Equation (15). Additional points are added between the decision variables in $\boldsymbol{f}_{\text {norm }}$. In total ten decision variables were used and with $N_{\text {ext }}$ equal to ten, a total of $N_{\text {inn }}=91$ force-slip points are used when fitting the tyre parameters in each of the inner optimization problems.

$$
\begin{gathered}
f_{\text {ref }, k, 1}(j)=\left(\boldsymbol{f}_{\text {norm }}(1)+\frac{\boldsymbol{f}_{\text {norm }}(2)-\boldsymbol{f}_{\text {norm }}(1)}{N_{\text {ext }}} *(j-1)\right) * \mu_{\text {ref }, k} \\
f_{r e f, k, 2}(j)=\left(\boldsymbol{f}_{\text {norm }}(2)+\frac{\boldsymbol{f}_{\text {norm }}(3)-\boldsymbol{f}_{\text {norm }}(2)}{N_{\text {ext }}} *(j-1)\right) * \mu_{\text {ref }, k} \\
\cdot \\
\cdot \\
\cdot \\
f_{r e f, k, N-1}(j)=\left(\boldsymbol{f}_{\text {norm }}(N-1)+\frac{\boldsymbol{f}_{\text {norm }}(N)-\boldsymbol{f}_{\text {norm }}(N-1)}{N_{\text {ext }}} *(j-1)\right) * \mu_{\text {ref }, k} \\
f_{r e f, k, N}=\boldsymbol{f}_{\text {norm }}(N) * \mu_{r e f, k}
\end{gathered}
$$


for

$$
j=1,2,3 \ldots N_{\text {ext }}
$$

The extra points that are added in between the decision variables, with the number of points determined by the parameter $N_{\text {ext }}$, provide two advantages. In order to decrease the computation time, the number of decision variables should be small. However, a small number of decision variables mean that the influence of the modelling errors potentially could be larger since two well-chosen measurement points can give a small friction estimate error. Two points could thus be chosen that would give large fitting errors in the inner optimization problem but result in a small friction estimation error. The additional points in between the decision variables remove the possibility to skip certain ranges of normalized tyre force. The extra data points in the inner optimization problem therefore reduce the likelihood to find data specific solutions due to modelling errors. Secondly, the cost function for the outer optimization problem should be smooth. The data with additional data points between the decision variables have a larger distribution since the cost function has to be evaluated in all these data points. This reduces the risk of the optimization algorithm getting stuck in a local minimum.

The inner problem of finding the tyre parameters given a certain excitation is solved using a trust-region-reflective algorithm with upper and lower bounds on the tyre parameters, table 1 . See [21] for a more extensive description of the algorithm. This algorithm was supplied with the Jacobian and Hessian of the objective function with respect to the decision variables to decrease the optimization time.

The objective function in the outer optimization problem is directly dependent on how well the tyre model in the inner optimization problems fit the reference data. Due to these tyre modelling errors the outer cost function may not change smoothly with changing force excitation. Furthermore, the outer optimization problem may have many local minima due to the dependence on the inner problem. Gradient based optimization algorithms are thus unsuitable and a genetic optimization algorithm is used instead. See [22] for a more extensive description of genetic algorithms. In the used genetic algorithm the children that are created through crossover and elite children are not mutated, see [23].

Stochastic optimization methods, including genetic algorithms, may converge to different solutions when running the algorithms repeatedly. This means that there is no guarantee that the global minimum is obtained. On the other hand, if several solutions have similar objective function values they are all viable solutions, only slightly worse than the optimal solution [22]. The genetic algorithm thus fulfils the need to find a good (with low cost) excitation strategy that can be used as inspiration for excitations that can be implemented in a real vehicle and as a benchmark to which other excitations strategies can be compared. The optimization was run several times for each investigated case in order to increase the chance of finding the global minimum.

\subsection{Suboptimal excitations}

The excitation found through the optimization problem relies on a-priori information of the maximum friction coefficient. It is thus not certain that it will be possible to implement the optimized tyre force excitation in practice since the actual tyre-road combination is unknown. This is naturally also the main motivation behind the active tyre force excitation.

Two excitation strategies that are straightforward to implement in a real vehicle, a ramp in the tyre force and a ramp in the slip ratio were evaluated and compared to the optimized excitation. These strategies have the benefit that they do not require knowledge of the maximum road friction coefficient, i.e. regardless of the actual maximum tyre-road friction coefficient the 
excitation strategies can be implemented. The sampled data will therefore correspond to the chosen estimation strategy even if the intervention has to be aborted, for instance due to safety reasons. In contrast, when using a nonlinear excitation strategy it is hard to predict at which utilization level the intervention will be stopped.

The last strategy that is evaluated assume a recursive tyre parameter estimation. This method thus has a slow ramp until a force threshold is reached at which the gradient is increased and held as long as the force is below the utilization limit. The force is then slowly decreased until the sample limit is reached, see Figure 5. The idea is to determine the stiffness quickly, increase the force until the maximum allowed utilization is reached and then decrease the force slowly to determine the curvature of the slip-force curve near the friction limit. Due to the purpose of the excitation, to determine the slip stiffness before the other tyre parameters, the excitation is referred to as stiffness ramp.

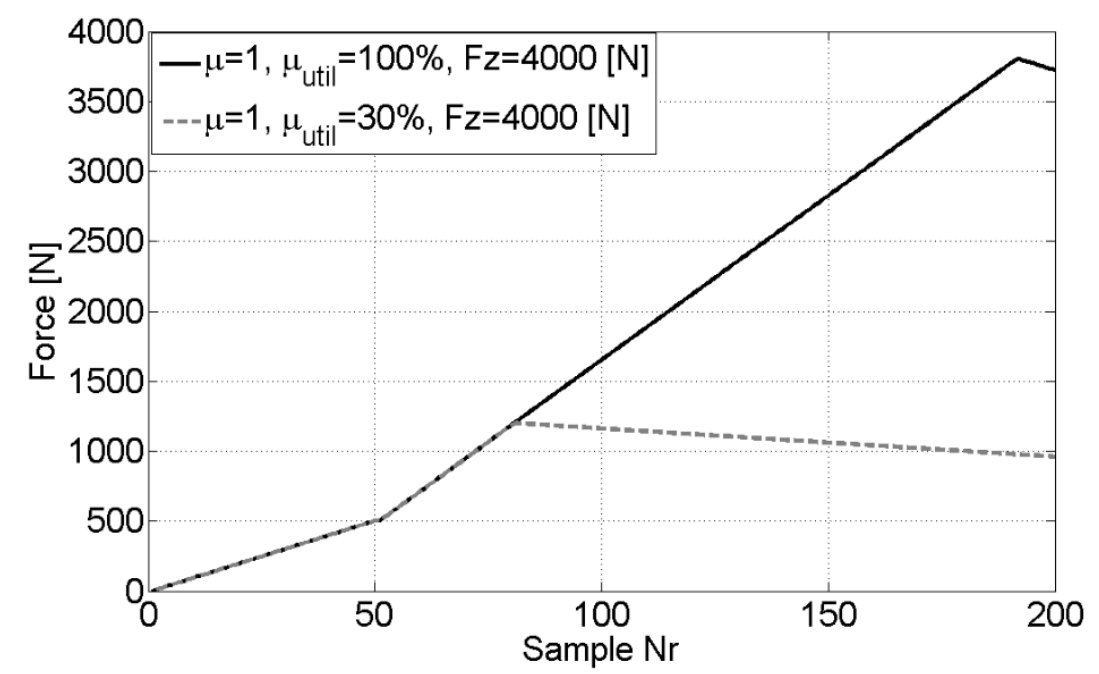

Figure 5, Excitation designed to first estimate the stiffness and then quickly reach large utilization, hereafter referred to as stiffness ramp. 


\section{Optimized Excitations}

This section presents the results from the optimizations and compare the estimation errors for different excitation strategies. First some general results are presented where the friction estimation errors for a force ramp are presented. The optimized excitations indicate the estimator performance that can be achieved for the investigated tyre-road combinations. Although the tyre-road combinations are chosen to have different tyre characteristics in order to make the results more general, the optimized excitations have not been validated for other tyre-road combinations than the ones used in the optimization. The optimized excitations are intended to be used as a benchmark for other excitations and as inspiration for other excitation strategies.

\subsection{Friction estimation error with a force ramp excitation}

To get a basic understanding of the friction estimation error for different utilization levels, the inner optimization problem was used to estimate the tyre parameters for a force ramp with increasing utilized friction $\mu_{u t i l}$ up to $95 \%$ friction utilization, see Figure 6.200 samples were used for all excitation levels. The friction estimates and the corresponding errors were normalized for easier interpretation. On the asphalt surface, the brush model with parabolic pressure distribution provides a reasonable friction estimate for all excitation levels. In comparison the Dugoff model tends to follow the utilized friction more closely. The magic tyre formula has a small estimation error for all utilization on gravel. However, on the asphalt surface the error increases for large excitations. Note that these results are valid for one of the three investigated tyres. Also note that the error is increasing for larger utilizations on both wet asphalt and gravel for the Magic Formula both with and without fixed $\mathrm{C}$ parameter.
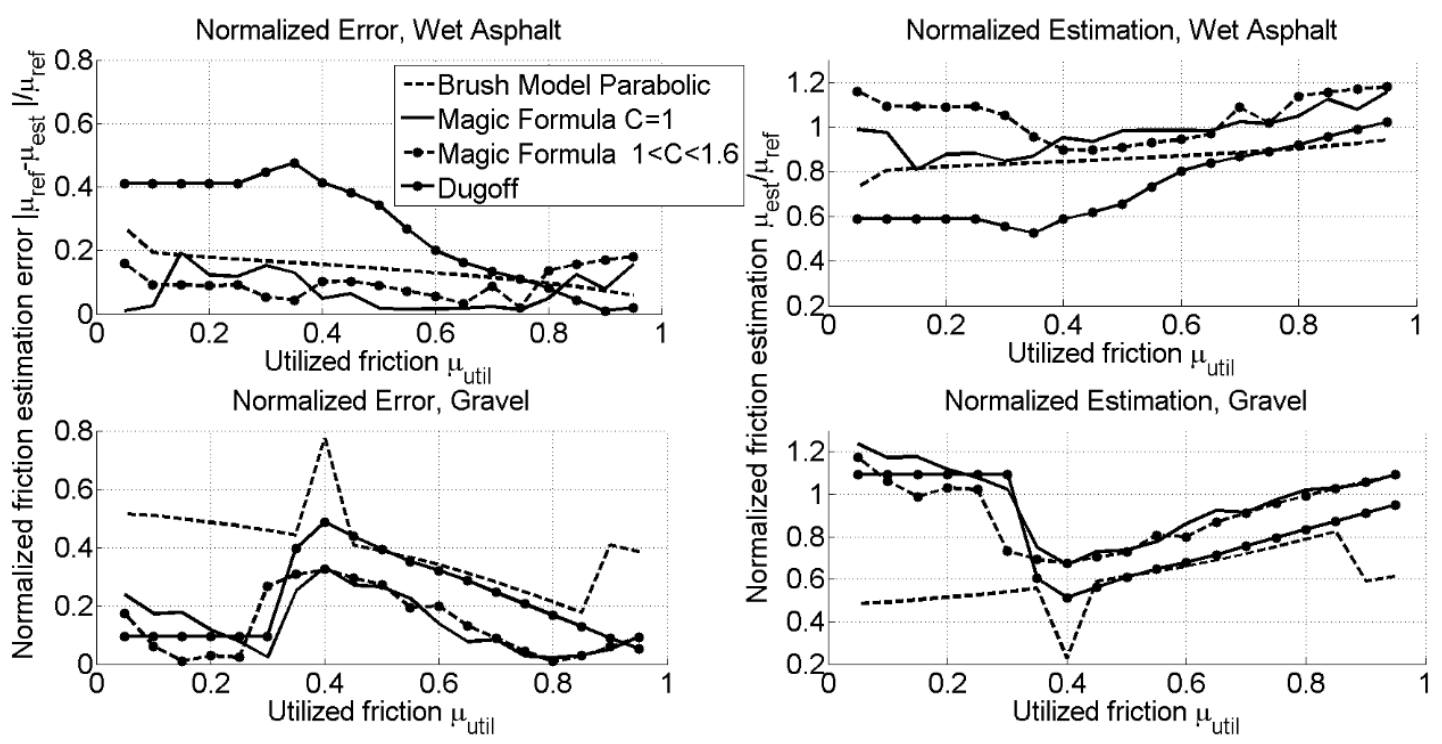

Figure 6, Normalized friction estimation error as a function of utilized friction for a force ramp excitation, winter tyre on wet asphalt and gravel for all tyre models.

\subsection{Optimized excitation for a given maximum tyre utilization}

\subsubsection{Optimized excitation for two road surfaces}

The results from three different optimization runs for each tyre model can be seen in Figure 79. The maximum utilization level is limited at three different levels to investigate the differences in estimation error and estimation strategies depending on the allowed excitation level. 
The structures and modelling errors of the three different tyre models that are evaluated makes the optimized excitation strategies different between the models. The brush model with parabolic pressure distribution requires large excitation levels to estimate the friction coefficient. Hence, the tyre force is increased fast until the maximum excitation level is reached. For the magic tyre formula, the maximum allowed excitation level is avoided consistently both for fixed and free $\mathrm{C}$ parameter. This is likely due to the increase in error for large excitation levels, as seen from Figure 6. With free $\mathrm{C}$ parameter the maximum friction utilization is $71 \%$, this model is therefore not evaluated when the maximum utilization limit is set to $75 \%$ in Figure 8. Note also that the friction estimation error based on the estimated magic tyre formula parameters is smaller for a maximum utilization limit of 0.65 when the $\mathrm{C}$ parameter can be chosen freely.

The excitation for the Dugoff tyre model has a large gradient up to between $40-50 \%$ utilization. This can be due to the structure of the Dugoff model, where the friction coefficient does not affect the shape of the curve below 50\% utilization, as also seen in Figure 4. Note also that for all tyre models, only a few samples are found in the linear tyre region, presumably due to the fact that the stiffness can be sufficiently determined from a few points in a noise free environment. If noise is added though, more samples may be required in the linear tyre region to estimate the slip stiffness accurately.

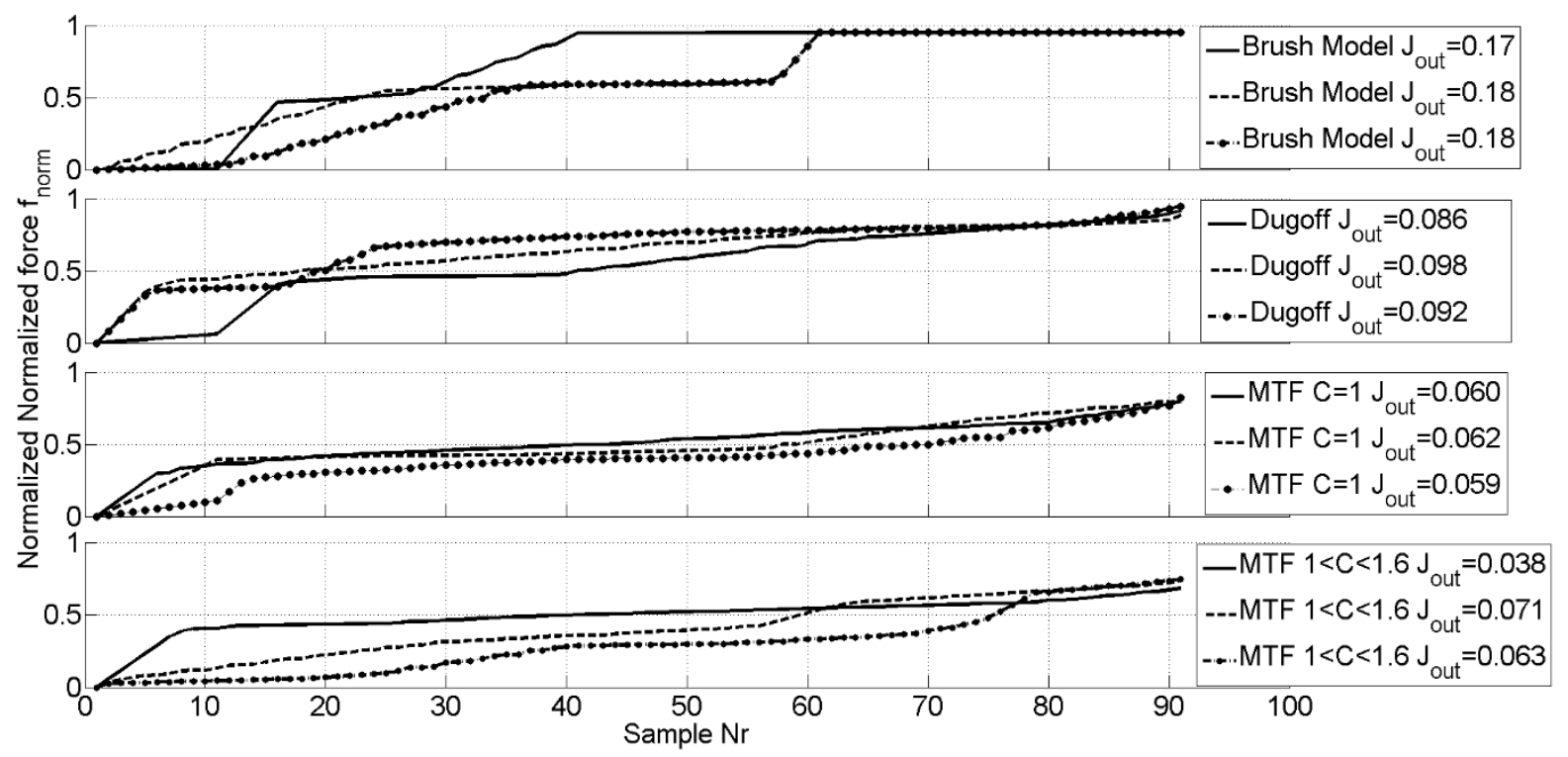

Figure 7, Optimized excitation for a maximum utilization of $95 \%\left(\mu_{u t i l, m a x}=0.95\right)$ for all road-tyre combinations 


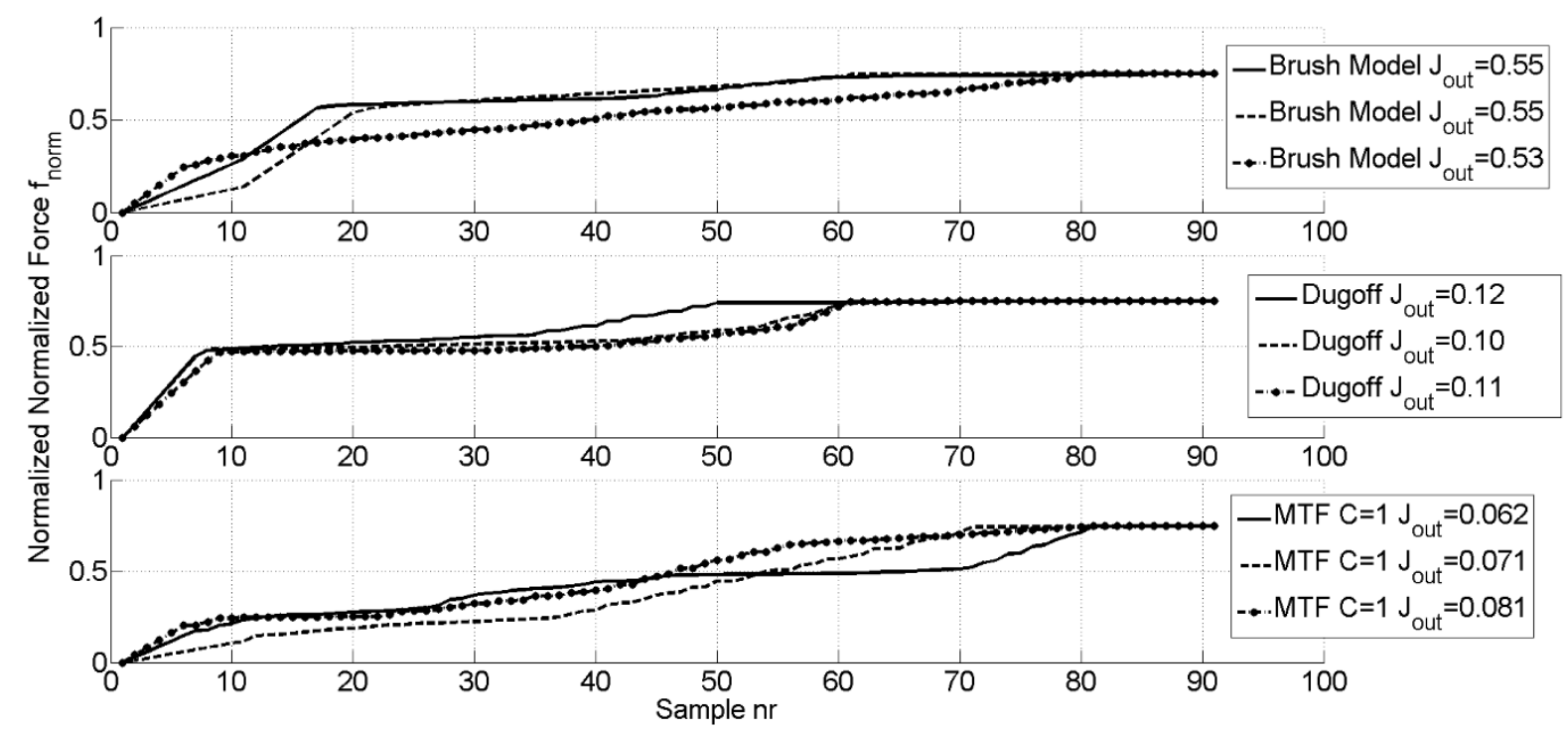

Figure 8, Optimized excitation for a maximum utilization of $75 \%\left(\mu_{u t i l, m a x}=0.75\right)$ for all road-tyre combinations, MTF with estimation of the parameter $C$ not included since the maximum utilization with $\mu_{u t i l, \max }=0.95$ was found to be $71 \%$.

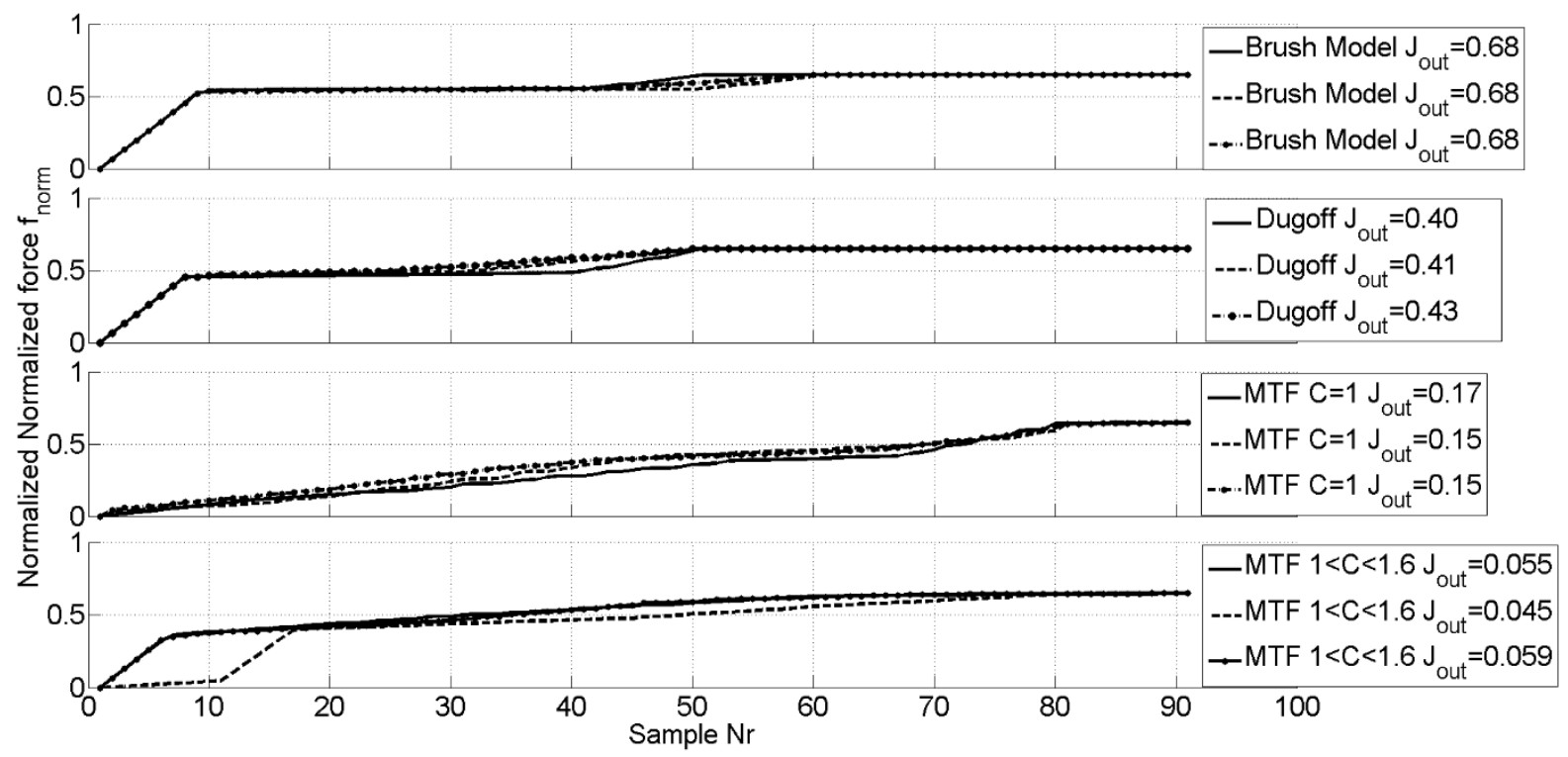

Figure 9, Optimized excitation for a maximum utilization of $65 \%\left(\mu_{u t i l, m a x}=0.65\right)$ for all road-tyre combinations,

\subsubsection{Optimized excitation for one road surface}

Due to the differences in tyre characteristics on gravel and wet asphalt, the modelling errors and hence the optimized excitation strategies differs between the surfaces, see Figure 10 and 11. For the brush model with parabolic pressure, the force is increased faster on asphalt than on gravel and the maximum utilization is consequently reached faster. The excitation strategy for the Dugoff model is shifted as well and the fast ramp in the beginning is not present on gravel for any of the optimized excitations. 


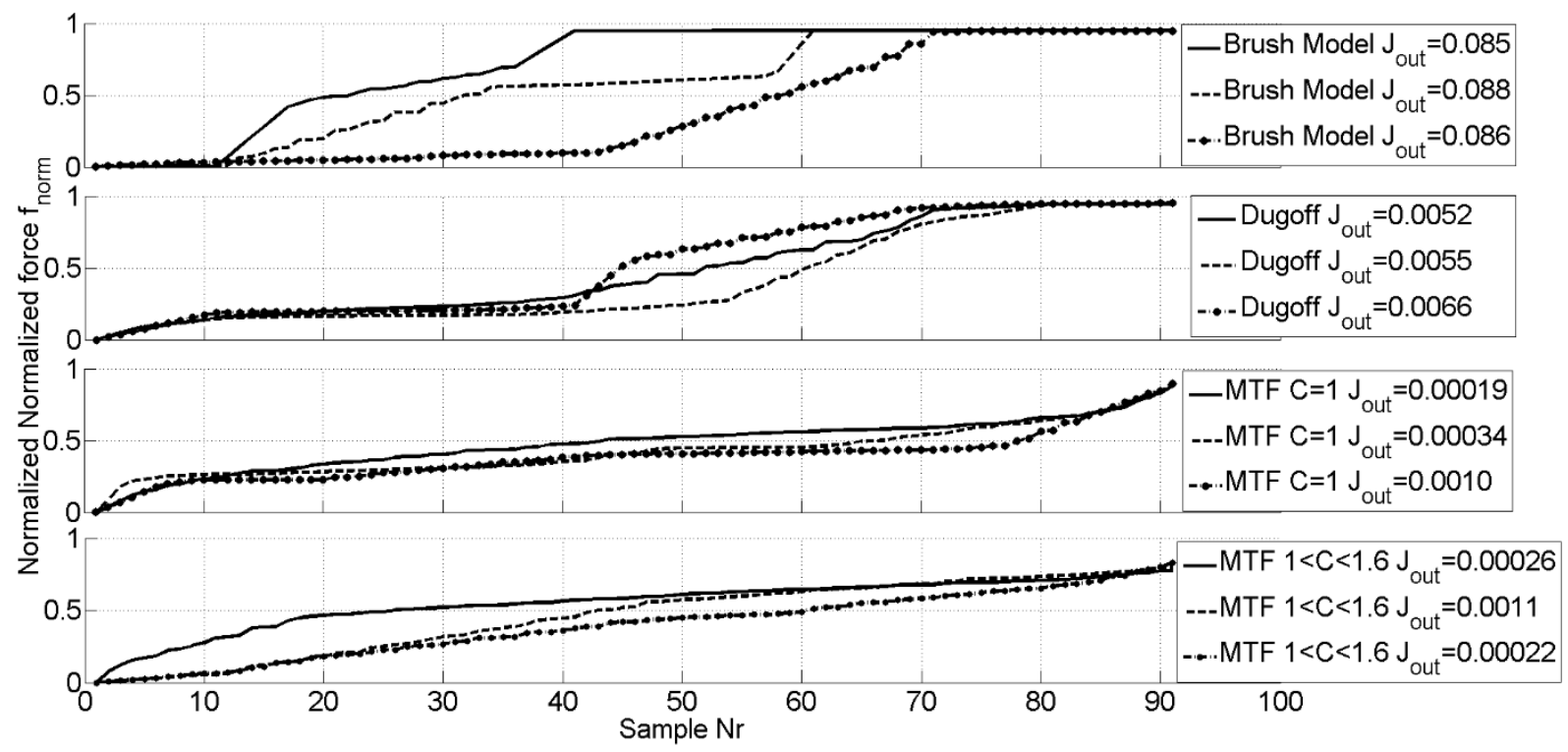

Figure 10, Optimized excitation for a maximum utilization of $95 \%\left(\mu_{u t i l \text { max }}=0.95\right)$ for all tyres on gravel.

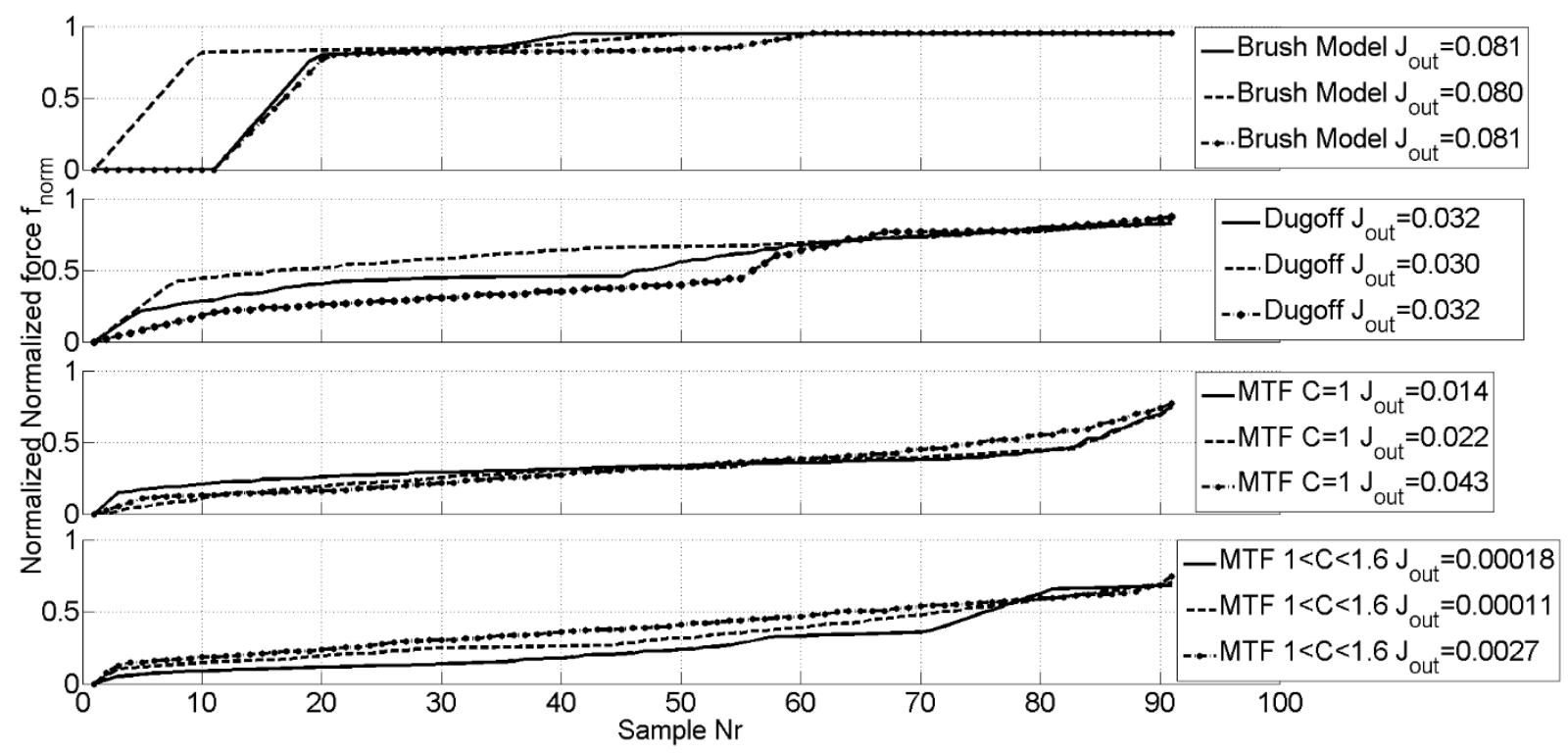

Figure 11, Optimized excitation for a maximum utilization of $95 \%\left(\mu_{u t i l, m a x}=0.95\right)$ for all tyres on wet asphalt.

\subsubsection{Excitation strategies based on optimized excitation}

The optimized excitation for the Brush model can be seen as a ramp with a steep slope to the maximum allowed utilization level. The tyre relaxation and physical constraints of the engine should be considered since they limit the maximum force gradient that can be used to achieve accurate measurement data. From [18], the maximum achievable torque gradient that the engine, for that particular vehicle, could deliver to the driven wheels was $1200 \mathrm{Nm} / \mathrm{s}$. Assuming a wheel radius of around 0.3, the maximum force gradient that can be used is $4000 \mathrm{~N} / \mathrm{s}$. This limit is hence used when implementing the saturated steep force ramp based on the optimized excitation for the brush model. The optimized strategies for the Magic formula and the Dugoff model are harder to implement in a way that would be neutral to all friction levels, i.e. the friction has to be known in order to implement these strategies. Although used as benchmarks when comparing different excitations they are not evaluated for all tyre utilizations. 


\section{Evaluation of different excitation strategies}

The different excitation strategies suggested in section 2.4 and 3.2.3 are evaluated and compared to the lowest cost function values obtained in the optimization. The slip ramp and the force ramp have a constant gradient in slip and force respectively, up to the maximum utilization level. The steep force ramp has a large constant force gradient until the force reaches the maximum utilization level. At this level the force is kept constant until the end of the intervention. The last evaluated excitation, the stiffness ramp, has a slow ramp at the beginning of the intervention, followed by a fast ramp up to the maximum utilization at which the force is slowly decreased, see Figure 5. The excitation strategies are evaluated both with and without measurement noise in the force and slip signals. The excitations are compared separately on the different surfaces and for all six tyre-road combination simultaneously.

\subsection{Estimation Error without noise}

The results for the different excitations evaluated on all six tyre-road combination simultaneously can be seen in Figure 12. Note that for both the Dugoff tyre model and the Brush model with parabolic pressure distribution, a saturated steep force ramp seem to be the best option. For the magic formula it is not as obvious which excitation strategy to use. Depending on the excitation level, the strategy with the lowest cost varies. Generally though, the magic formula has a smaller error than the two other tyre models. Note that the magic formula with fitted $\mathrm{C}$ parameter does not obtain as low cost function values as found for the optimized excitation. The difference between fitting the $\mathrm{C}$ parameter or not is hence smaller when a suboptimal excitation is used. A possible explanation is the fact that a larger number of parameters allow the outer optimization problem to adjust the excitation in a way which gives a small friction estimation error for that particular excitation level.
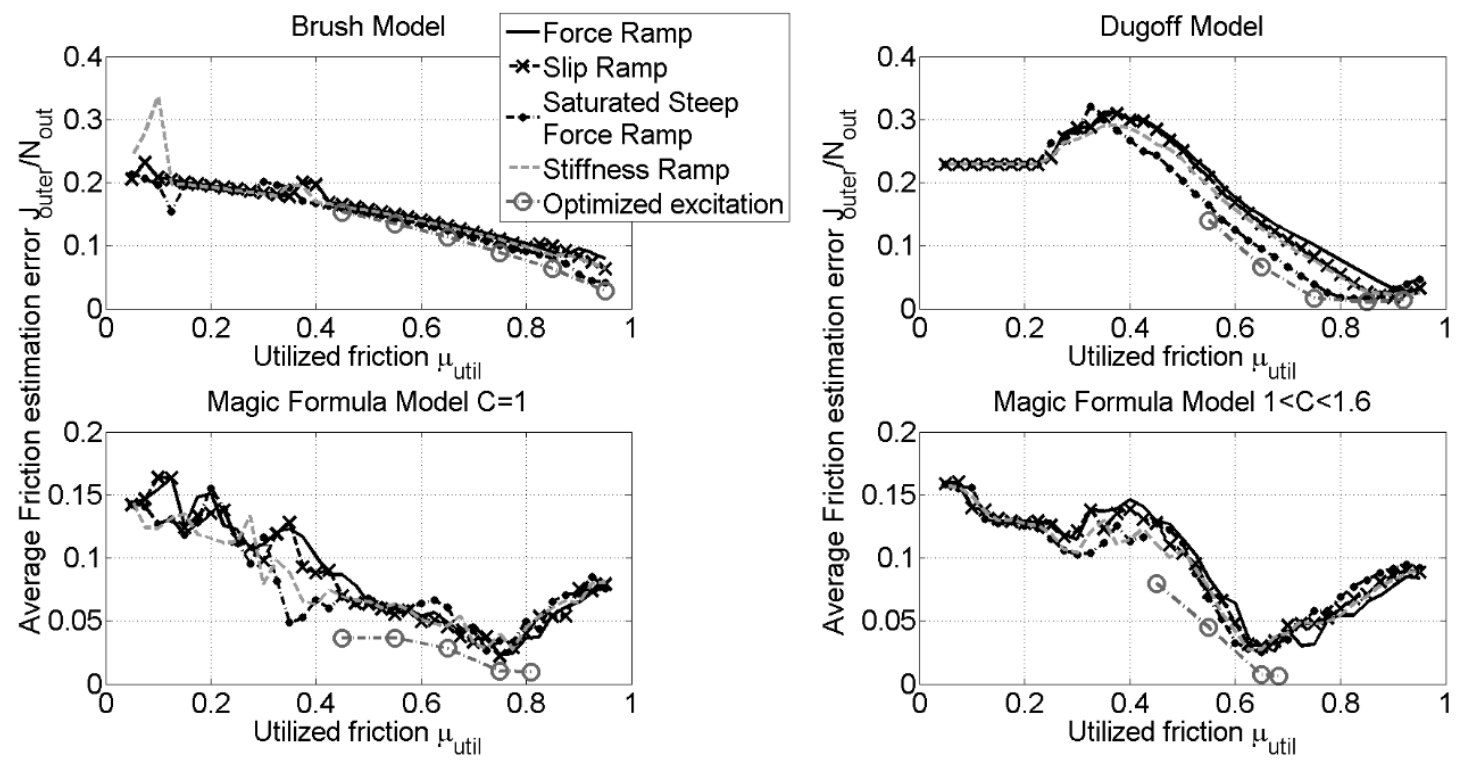

Figure 12, Average friction estimation error $\frac{J_{\text {outer }}}{N_{\text {out }}}$ for all tyre-road combinations as a function of utilized friction for different excitation strategies, without any measurement noise

Due to differences in the tyre-road interaction, the modelling errors of the tyre models will be different on different road surfaces. It is thus not obvious if the same excitation strategy can be used on two different surfaces for the same tyre model. However, comparing Figure 12 with Figure 13-14, it seems that the saturated steep force ramp is the best of the evaluated excitation strategies on both road surfaces for the brush model and the Dugoff model. The Magic formula 
shows no clear indication of any excitation strategy that give a low error estimation error for all excitation levels on either surface.
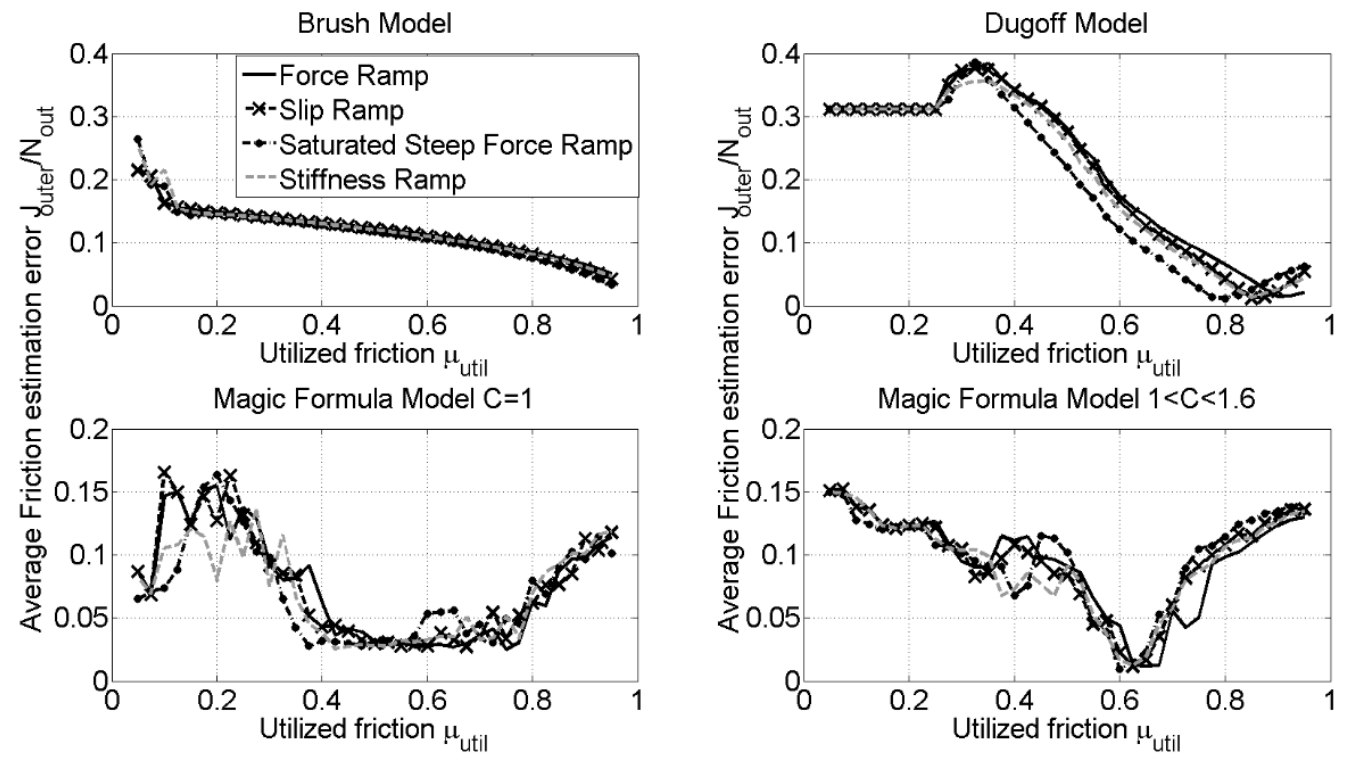

Figure 13, Average friction estimation error $\frac{J_{o u t e r}}{N_{\text {out }}}$ for all tyres on wet asphalt as a function of utilized friction for different excitation strategies, without any measurement noise
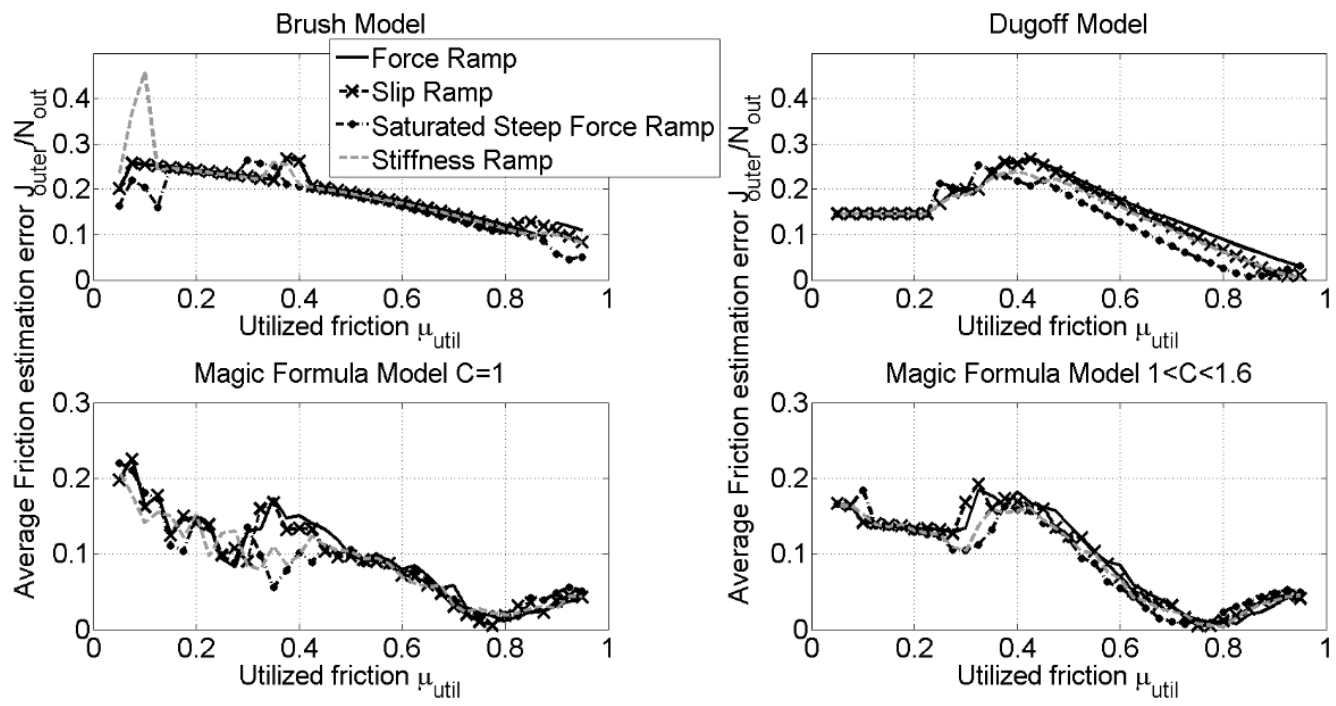

Figure 14, Average friction estimation error $\frac{J_{\text {outer }}}{N_{\text {out }}}$ for all tyres on gravel as a function of utilized friction for different excitation strategies, without any measurement noise

\subsection{Estimation error with noise}

When implementing a recursive estimator in real time on-board a vehicle there will be noise and disturbances on the signals used to estimate both the force and the slip ratio. Noise should thus be included in the evaluation to make the comparison more realistic. The noise is modelled as zero mean white Gaussian noise with a variance of $0.03^{2}$ for the force signal $f$ and $0.003^{2}$ for the slip ratio signal $\sigma_{x}$, these noise levels approximately correspond to the ones in previous experiments, see [18]. The excitation strategies are evaluated 30 times each for each tyre model and the mean friction estimation error is calculated, see Figure 15-17. 
With the added noise the saturated steep force ramp is not the best excitation strategy for the Brush model anymore, see Figure 15. Instead the basic force ramp is the best option for the Brush model and both Magic formula models in the medium excitation range. For the Dugoff model though, the saturated steep force ramp remains the best choice for friction utilizations between approximately $0.3-0.85$. Note the significantly larger mean error for both magic formulas when noise is present. Furthermore, the noise changes the shape of the curve to a more or less monotonically decreasing error with increasing utilization. The result from the different road surfaces, Figure $16 \& 17$, confirm the results in Figure 15.
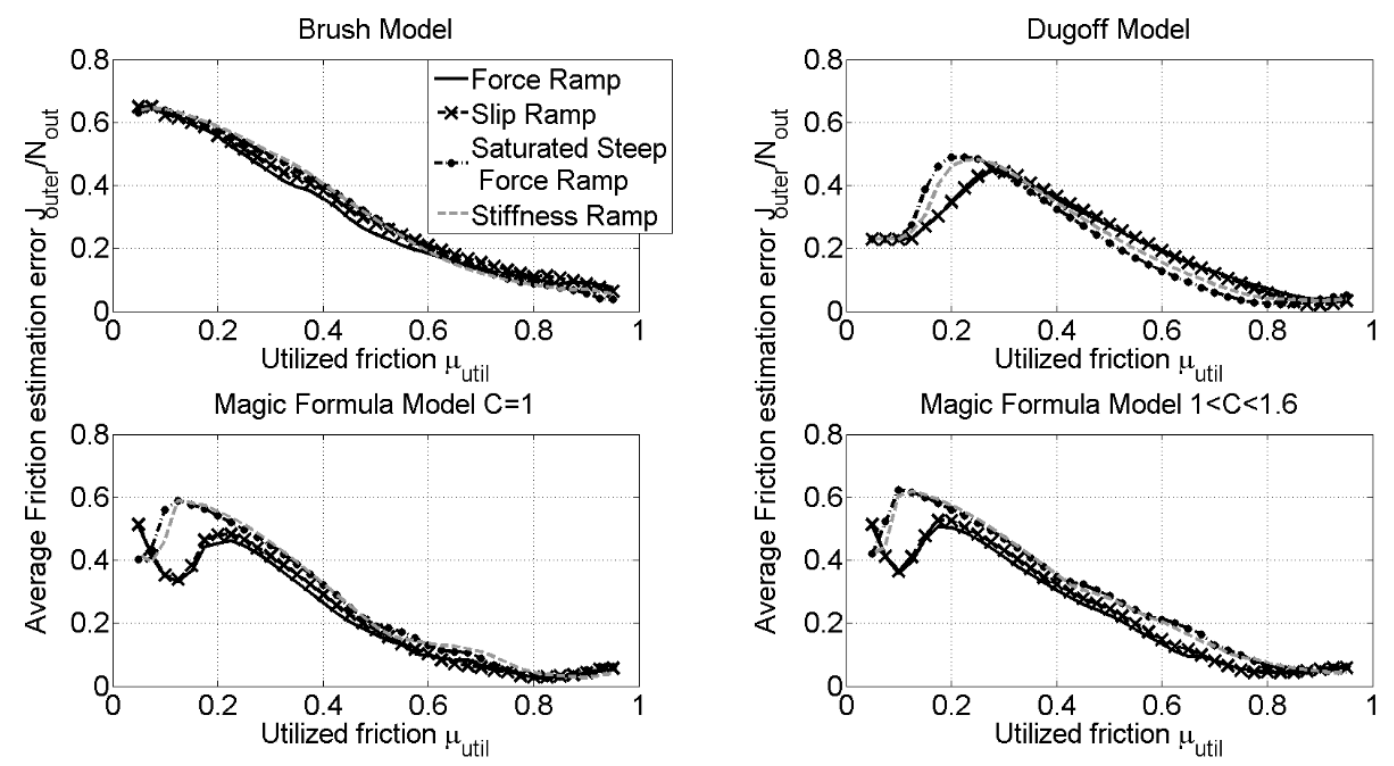

Figure 15, Average friction estimation error $\frac{J_{o u t e r}}{N_{\text {out }}}$ for all tyre-road combinations as a function of utilized friction for different excitation strategies, noise added to the slip and force signal.
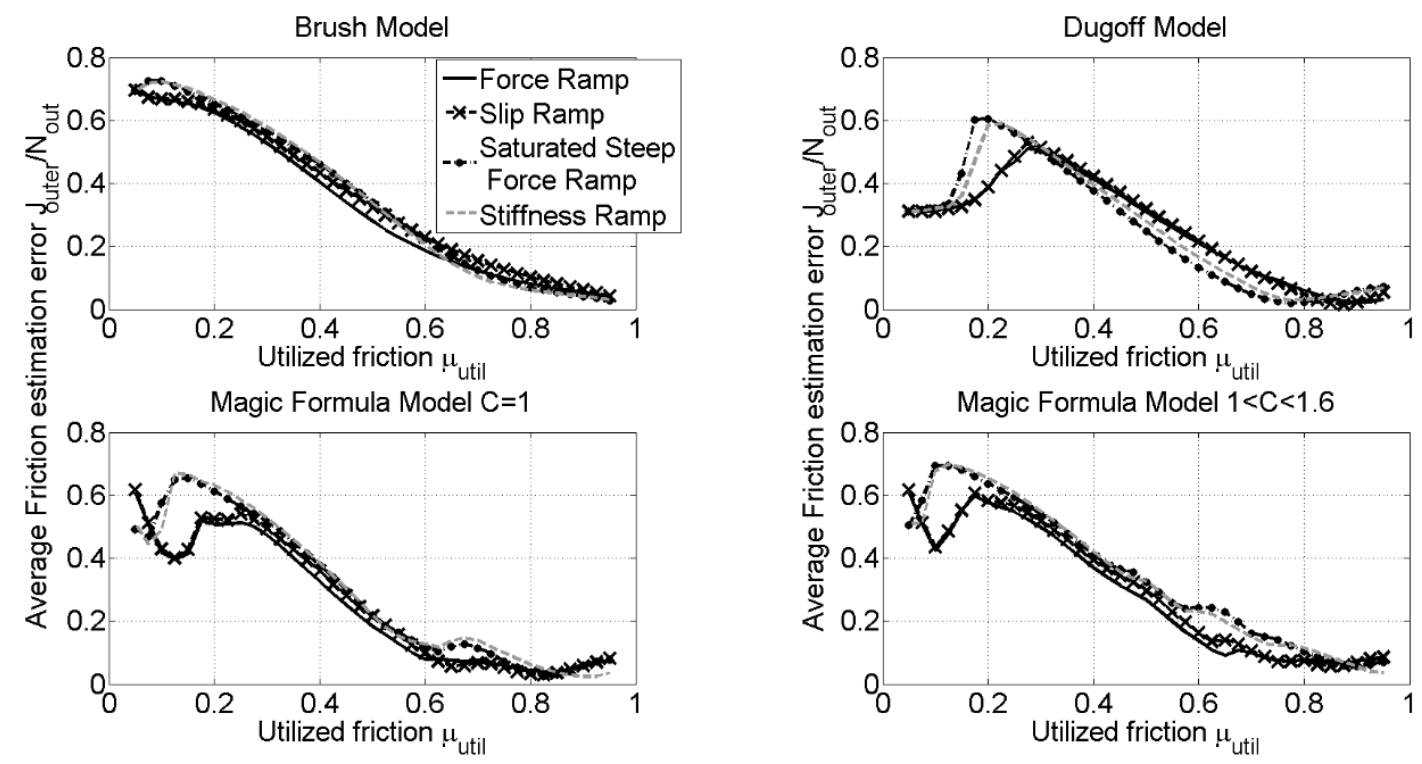

Figure 16, Average friction estimation error $\frac{J_{\text {outer }}}{N_{\text {out }}}$ for all tyres on wet asphalt as a function of utilized friction for different excitation strategies, noise added to the slip and force signal. 

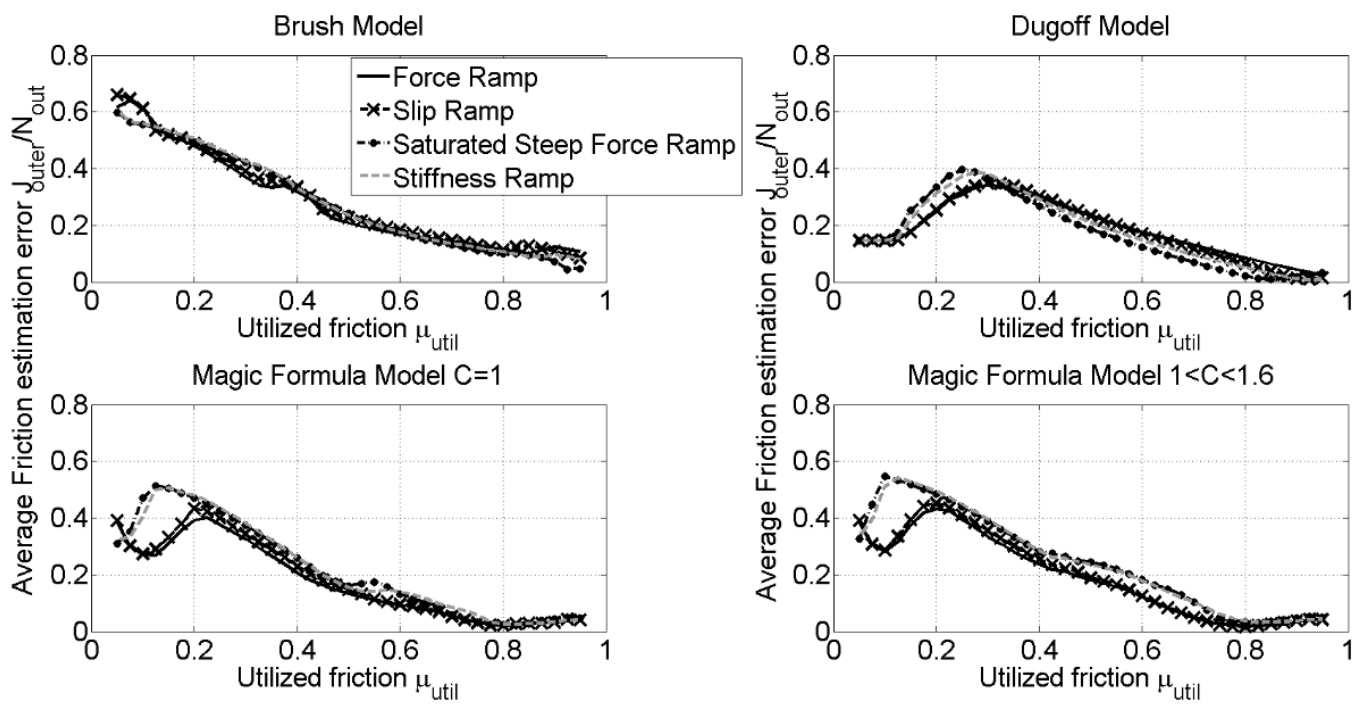

Figure 17, Average friction estimation error $\frac{J_{\text {outer }}}{N_{\text {out }}}$ for all tyres on gravel as a function of utilized friction for different excitation strategies, noise added to the slip and force signal. 


\section{Discussion}

\subsection{C parameter in the Magic Formula}

For the optimized excitation, a larger friction estimation error was found for the Magic formula with fixed $\mathrm{C}$ parameter compared to when $\mathrm{C}$ was estimated. This does not necessarily mean that estimating the shape factor reduces the estimation error for a more realistic excitation compared to using a fixed value. With the additional parameter, the genetic algorithm has a larger possibility to take advantage of the modelling errors and choose a specific excitation strategy which minimizes the friction estimate error. The comparisons between the implementable excitation strategies are therefore more representative for a real vehicle.

When comparing the two Magic formula models in Figure 12-14, no clear difference in the size of the estimation errors can be seen. If anything, the Magic formula with fixed $\mathrm{C}$ parameter has a flatter error curve with lower estimation error in the medium excitation ranges. If estimating the $\mathrm{C}$ parameter does not lower the estimation error, it should not be included in the estimator since that would unnecessarily increase the number of parameters. However, the evaluation should be performed for more types of road surfaces and for more tyres to increase the generality of the results.

\subsection{Optimized excitation for a given maximum tyre utilization}

None of the evaluated excitation strategies provide the lowest error for all tyre utilizations. However, for the Dugoff tyre model the saturated steep force ramp is a strong candidate. For the medium excitation levels between 0.3-0.8 this excitation strategy had the lowest error. Due to the linear part of the tyre characteristics, it should not be expected that the friction coefficient can be estimated at a utilization level below 0.3 . Furthermore, this excitation strategy has fairly similar error as the other strategies at utilization above 0.8 .

For the other tyre models the differences between the excitation strategies are smaller. The saturated steep force ramp produces the lowest error for the brush model without any measurement noise but with added noise the force ramp seems to be the best option overall, see Figure 15. The Magic Formula performs well without any measurement noise and it is difficult to say which excitation strategy that perform best. By adding noise, the force ramp becomes the stronger candidate for the Magic formula both with and without locked $\mathrm{C}$ parameter.

The noise does not only affect which excitation that give the lowest error but also the size of the estimation error. For the brush model and the Dugoff model, the trend with a decreasing estimation error for an increasing utilization is valid both with and without noise. For the Magic formula though, the estimation error varies across the utilization range without noise but at a consistently low value. When noise is added, the Magic Formula displays the same trend as the brush model and the Dugoff model. This can partly be explained by the cost function of the inner estimation problem that minimizes the force error between the model and the data. When the noise on the slip signal is too large, the data points can be interpreted as data points from a low-friction surface and the tyre parameters are thus fitted accordingly.

\subsection{Minimum tyre utilization required to achieve less than an estimation error of less than 0.1}

The minimum tyre utilization required to achieve an estimation error of less than 0.1 without any measurement noise is shown in table 2 . An estimation error of 0.1 roughly corresponds to an error of $12-14 \%$ on wet asphalt and $13-22 \%$ on gravel (Figure 1). As seen from the table the Magic formula has a smaller required utilization compared to the other two tyre models. Furthermore, the Magic formula with a fixed $\mathrm{C}$ parameter has a lower required utilization for 
the suboptimal excitations that were investigated. For these tyre-road combinations the best performing tyre model only requires around 30\% utilization to have an average estimation error of below 0.1. Of course, in individual cases the estimation error can be larger. Both the Dugoff model and the Brush model achieve similar performance with measurement noise, compare table 2 and 3. However, the required utilization for the Magic tyre formula increases substantially when measurement noise is added and the performance is decreased to a level close to the Dugoff model. Minimizing the measurement noise is thus of importance when the performance of the friction estimator should be improved.

Table 2, Required utilization for a friction estimation error of less than 0.1 without any measurement noise, from Fig. 12

\begin{tabular}{|l|c|}
\hline \multicolumn{1}{|c|}{ Tyre Model } & $\begin{array}{c}\text { Required Utilization [\%], } \\
\text { Optimized excitation/Other } \\
\text { excitation }\end{array}$ \\
\hline Brush Model & $71 / 77.5$ \\
\hline Dugoff Model & $60 / 64.5$ \\
\hline Magic formula, $1<\mathrm{C}<1.6$ & $<45 / 51$ \\
\hline Magic formula, $\mathrm{C}=1$ & $<45 / 29$ \\
\hline
\end{tabular}

Table 3, Required utilization for a friction estimation error of less than 0.1 with measurement noise, from Fig. 15

\begin{tabular}{|l|c|}
\hline \multicolumn{1}{|c|}{ Tyre Model } & $\begin{array}{c}\text { Required Utilization [\%] } \\
\text { Other excitation }\end{array}$ \\
\hline Brush Model & 75 \\
\hline Dugoff Model & 67.5 \\
\hline Magic formula, $1<\mathrm{C}<1.6$ & 65 \\
\hline Magic formula, $\mathrm{C}=1$ & 60 \\
\hline
\end{tabular}

\subsection{Tyre Models and Tyre modelling errors}

The brush model and the Dugoff tyre model have two parameters. The Magic formula on the other hand has four parameters, or three in the case of a fixed $\mathrm{C}$ value. With only two parameters the tyre characteristics cannot be described accurately at all slip values and the structure of the tyre model will affect how well the tyre characteristics can be fitted on different surfaces. Since the friction coefficient estimate is based on a tyre model fitted to the measurement data at the current utilized friction, any modelling errors will affect the estimate. For instance, in Figure 13 and 11 it is apparent that the brush model has a lower average error on wet asphalt than on gravel.

Even though the Magic formula has additional parameters to describe the tyre characteristics, modelling errors will still be present. However, as seen in Figure 12-14 the Magic formula has a lower error than the brush model and the Dugoff tyre model in a noise free environment. Based on these results, it appears beneficial to include additional parameters in the tyre model, in addition to the slip stiffness and the maximum friction coefficient. However, the same number of samples has been used for each utilization level and the measurements points have been chosen by the excitation strategy in a well-defined intervention. Without active tyre force excitation, the driver controls the manoeuvre in which the friction coefficient is estimated and the measurement points will hence have a more uneven distribution. For this type of data it can be beneficial to have fewer parameters. The tyre model should therefore be chosen with the expected excitation in mind. 
It is also interesting to note that the Magic formula gives the lowest estimation error below $80 \%$ utilized friction on both wet asphalt and gravel, although the estimation error does not increase as much above $80 \%$ on gravel as on wet asphalt. This indicate that the prediction made by the Magic formula based on the measurement points is incorrect, likely due to modelling errors. Of course, the level of excitation cannot be chosen freely in a real vehicle since the friction coefficient is unknown. It is thus important to investigate how the error varies across all excitation levels and not only the minimum error. 


\section{Conclusions \& Future Work}

This paper investigated different excitation strategies for tyre-road friction estimation using active tyre force excitation. Optimized excitations were compared to other excitations that can be implemented in real-time in a vehicle with limited information about the road surface.

Without any measurement noise, the Magic formula, which has the most number of parameters, showed the most promising results of the investigated tyre models. At realistic noise levels, the benefits of additional parameters are smaller and the simpler tyre models can thus be used. Ideally the measurement signals needed for friction estimation should have low noise and small disturbances in order to decrease the friction estimation error. Carefully estimating these signals is thus important for the performance of the estimator and should be given time in the tyre-road friction estimator development process.

It was found that different excitation strategies lead to the lowest friction estimation error depending on the choice of tyre model in the estimator. The choice of tyre model should thus be taken into consideration when designing an excitation strategy. The sensitivity to different excitation strategies varied between the tyre models with some tyre models being less sensitive. This can be considered a benefit since an excitation strategy that suits other requirements such as safety, comfort and wear can be chosen. For the Magic formula, which had the best performance of the investigated tyre models, a simple force ramp seems to be the most promising excitation strategy. Without any measurement noise this tyre model showed no clear preferred excitation strategy but with added measurement noise, the force ramp provided the best estimator performance. The force ramp has the benefit of being easily implementable in real-time. In order to have an average estimation error of below 0.1 in normalized force with realistic noise levels and with the force ramp excitation, at least $60 \%$ utilization is required for the given tyre-road combinations. However, depending on the tyre-road combination, the required utilization varied between 40 and $65 \%$ in a noise free environment. Hence, without any measurement noise, $65 \%$ friction utilization will give a friction estimation error of below 0.1 in normalized force for all of the tested tyre-road combinations using a real-time implementable excitation strategy.

This paper has investigated how the force should be applied in an active tyre force intervention to minimize the friction estimation error. As such, it provides a basic understanding of how large variations that can be expected in estimator performance from different excitation strategies and when using different tyre models. If these kinds of systems are to be implemented in production vehicles, other aspects such as safety, comfort, and wear should be taken into account as well. The proposed method can be extended to include these aspects as well but would require the availability of the control algorithm with realistic actuator models, a complex vehicle simulation model and a model of the tyre wear. Future work should also include a recursive excitation strategy that is based on the current friction estimate and utilizes the information from the sensitivity analysis performed in the paper.

\section{Acknowledgements}

This work was supported by VINNOVA (the Swedish Governmental Agency for Innovation Systems) under grant number 2012-03658. 


\section{References}

[1] "Taxonomy and Definitions for Terms Related to On-Road Motor Vehicle Automated Driving Systems, J3016_201401," ed: SAE International, 2014.

[2] C. Ahn, H. Peng, and H. E. Tseng, "Robust estimation of road frictional coefficient," Control Systems Technology, IEEE Transactions on, vol. 21, pp. 1-13, 2013.

[3] M. R. Uchanski, "Road friction estimation for automobiles using digital signal processing methods," University of California, Berkeley, 2001.

[4] J. Casselgren, S. Rosendahl, and J. Eliasson, "Road surface information system," in Proceedings of the 16th SIRWEC conference: Helsinki, Finland (23-25th May 2012), 2012.

[5] (2016, 2016-06-02). Road Status Information. Available: http://www.roadstatus.info/about-rsi/

[6] U. Sandberg, "Influence of road surface texture on traffic characteristics related to environment, economy and safety: A state-of-the-art study regarding measures and measuring methods," VTI, Statens väg och transportforskningsinstitut VTI notat 53A1997, 1998.

[7] C. Lex, "Estimation of the Maximum Coefficient of Friction between Tire and Road Based on Vehicle State Measurements," $\mathrm{PhD}$ dissertation, Graz University of Technology, 2015.

[8] J. Svendenius, "Tire modeling and friction estimation," Lund University, 2007.

[9] W. R. Pasterkamp and H. Pacejka, "The tyre as a sensor to estimate friction," Vehicle System Dynamics, vol. 27, pp. 409-422, 1997.

[10] L. R. Ray, "Nonlinear tire force estimation and road friction identification: simulation and experiments," Automatica, vol. 33, pp. 1819-1833, 1997.

[11] M. J. Matilainen and A. J. Tuononen, "Tire friction potential estimation from measured tie rod forces," in Intelligent Vehicles Symposium (IV), 2011 IEEE, 2011, pp. 320-325.

[12] F. Gustafsson, "Slip-based tire-road friction estimation," Automatica, vol. 33, pp. 10871099, 1997.

[13] D. Pavković, J. Deur, J. Asgari, and D. Hrovat, "Experimental analysis of potentials for tire friction estimation in low-slip operating mode," SAE Technical Paper 0148-7191, 2006.

[14] C. R. Carlson and J. C. Gerdes, "Consistent nonlinear estimation of longitudinal tire stiffness and effective radius," Control Systems Technology, IEEE Transactions on, vol. 13, pp. 1010-1020, 2005.

[15] Y. Chen and J. Wang, "Adaptive vehicle speed control with input injections for longitudinal motion independent road frictional condition estimation," Vehicular Technology, IEEE Transactions on, vol. 60, pp. 839-848, 2011.

[16] S. Matsumoto, T. Kimura, T. Takahama, and H. Toyota, "Road surface friction coefficient estimating apparatus," ed: Google Patents, 2002.

[17] J. Ekmark and J. Jansson, "A method and a computer readable storage device for estimating tire-to-road friction " EP Patent EP1481861 B1, 2007. 
[18] A. Albinsson, F. Bruzelius, T. Gustafsson, M. Jonasson, and B. Jacobson, "Identification of tyre characteristics using active force excitation," in 24th International Symposium on Dynamics of Vehicles on Roads and Tracks, Graz, Austria, 2015.

[19] H. Dugoff, P. S. Fancher, and L. Segel, "Tire performance characteristics affecting vehicle response to steering and braking control inputs," Highway Safety Research Institute, Ann Arbor, Michigan1969.

[20] E. Bakker, L. Nyborg, and H. B. Pacejka, "Tyre modelling for use in vehicle dynamics studies," SAE Technical Paper1987.

[21] Mathworks, "Equation Solving Algorithms," in Matlab R2015b Documentation, Optimization Toolbox, Systems of Nonlinear Equations, ed, 2015.

[22] M. Wahde, Biologically inspired optimization methods: an introduction: WIT press, 2008

[23] Mathworks, "How the Genetic Algorithm Works," in Matlab R2015b Documentation, Global Optimization Toolbox, Genetic Algorithm, ed, 2015. 
Table 1, Tyre Parameter Constraints

\begin{tabular}{|l|l|c|l|}
\hline$C_{\sigma_{x}}$ & {$[1,200]$} & $\mathrm{B}$ & {$[1,200]$} \\
\hline$\mu$ & {$[0.05,1.5]$} & $C$ & {$[1,1]$ or $[1,1.6]$} \\
\hline$D$ & {$[0.05,1.5]$} & $E$ & {$[-1.5,1]$} \\
\hline
\end{tabular}


Table 2, Required utilization for a friction estimation error of less than 0.1 without any measurement noise, from Fig. 12

\begin{tabular}{|l|c|}
\hline \multicolumn{1}{|c|}{ Tyre Model } & $\begin{array}{c}\text { Required Utilization [\%], } \\
\text { Optimized excitation/Other } \\
\text { excitation }\end{array}$ \\
\hline Brush Model & $71 / 77.5$ \\
\hline Dugoff Model & $60 / 64.5$ \\
\hline Magic formula, $1<\mathrm{C}<1.6$ & $<45 / 51$ \\
\hline Magic formula, $\mathrm{C}=1$ & $<45 / 29$ \\
\hline
\end{tabular}


Table 3, Required utilization for a friction estimation error of less than 0.1 with measurement noise, from Fig. 15

\begin{tabular}{|l|c|}
\hline \multicolumn{1}{|c|}{ Tyre Model } & $\begin{array}{c}\text { Required Utilization [\%] } \\
\text { Other excitation }\end{array}$ \\
\hline Brush Model & 75 \\
\hline Dugoff Model & 67.5 \\
\hline Magic formula, $1<\mathrm{C}<1.6$ & 65 \\
\hline Magic formula, $\mathrm{C}=1$ & 60 \\
\hline
\end{tabular}




\section{Figure Captions}

Figure 1, Flow chart of optimization method

Figure 2, Measured and filtered normalized tyre force $\mathrm{f}$ and normalized normalized tyre force fnorm vs longitudinal slip ratio for the different tyre-road combinations.

Figure 3, Parameter sensitivity Magic formula, 0.1 normalized force variation.

Figure 4, Parameter sensitivity Brush model and Dugoff model, 0.1 normalized force variation.

Figure 5, Excitation designed to first estimate the stiffness and then quickly reach large utilization, hereafter referred to as stiffness ramp.

Figure 6, Normalized friction estimation error as a function of utilized friction for a force ramp excitation, winter tyre on wet asphalt and gravel for all tyre models.

Figure 7, Optimized excitation for a maximum utilization of $95 \%$ ( $\mu$ util, $\max =0.95)$ for all road-tyre combinations

Figure 8, Optimized excitation for a maximum utilization of $75 \%$ ( $\mu$ util, $\max =0.75$ ) for all road-tyre combinations, MTF with estimation of the parameter $\mathrm{C}$ not included since the maximum utilization with $\mu$ util, $\max =0.95$ was found to be $71 \%$.

Figure 9, Optimized excitation for a maximum utilization of $65 \%$ ( $\mu$ util, $\max =0.65$ ) for all road-tyre combinations,

Figure 10, Optimized excitation for a maximum utilization of 95\% ( $\mu$ util, max $=0.95$ ) for all tyres on gravel.

Figure 11, Optimized excitation for a maximum utilization of $95 \%$ ( $\mu$ util, max $=0.95$ ) for all tyres on wet asphalt.

Figure 12, Average friction estimation error Jouter/Nout for all tyre-road combinations as a function of utilized friction for different excitation strategies

Figure 13, Average friction estimation error Jouter/Nout for all tyres on wet asphalt as a function of utilized friction for different excitation strategies

Figure 14, Average friction estimation error Jouter/Nout for all tyres on gravel as a function of utilized friction for different excitation strategies

Figure 15, Average friction estimation error Jouter/Nout for all tyre-road combinations as a function of utilized friction for different excitation strategies, noise added to the slip and force signal.

Figure 16, Average friction estimation error Jouter/Nout for all tyres on wet asphalt as a function of utilized friction for different excitation strategies, noise added to the slip and force signal.

Figure 17, Average friction estimation error Jouter/Nout for all tyres on gravel as a function of utilized friction for different excitation strategies, noise added to the slip and force signal. 